\title{
Consommation de substances psychoactives des étudiants universitaires libanais : prévalence et facteurs associés
}

P. Salameh, ${ }^{1,2,3}$ S. Rachidi, ${ }^{1,2}$ A. Al-Hajje, ${ }^{1,2}$ S. Awada, ${ }^{1,2}$ K. Chouaib, ${ }^{2}$ N. Saleh ${ }^{2,3}$ et W. Bawab ${ }^{1,2}$

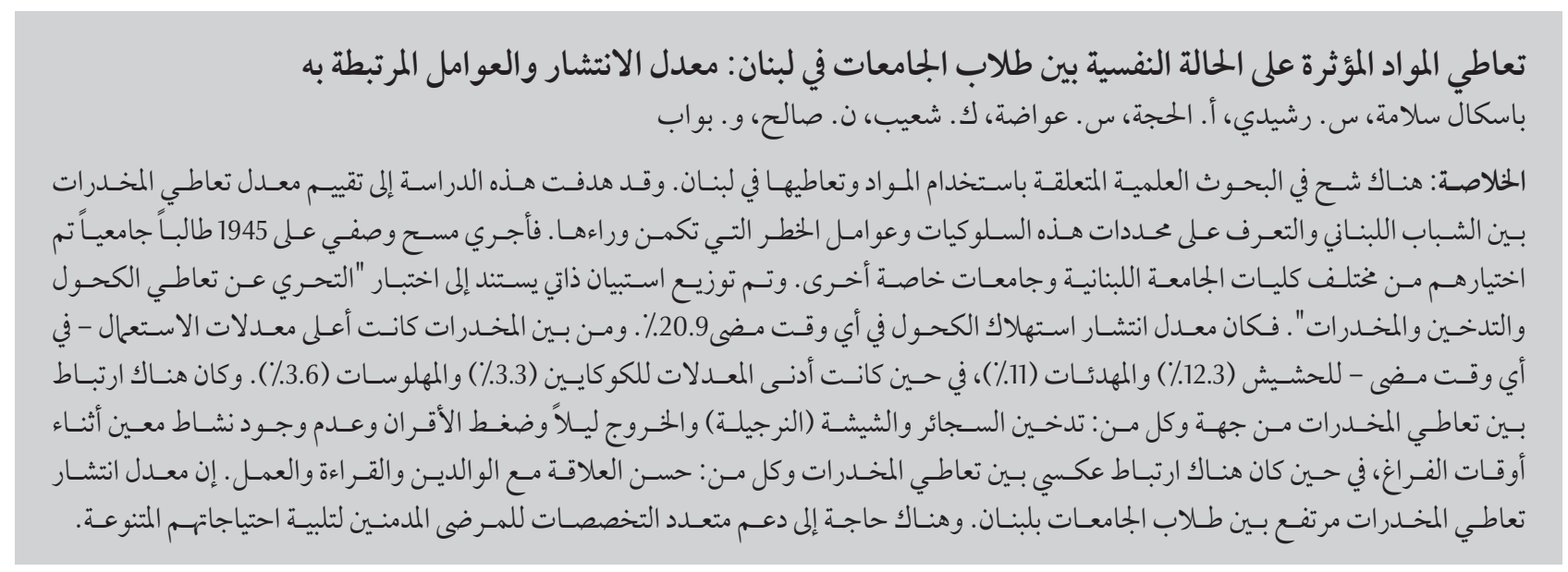

RÉSUMÉ Les études scientifiques sur la consommation et l'abus de substances psychoactives au Liban sont rares. Le but de cette étude était d'évaluer le taux de consommation et d'abus de ces substances auprès des jeunes Libanais et d'identifier les déterminants et facteurs de risque de tels comportements. II s'agit d'une étude observationnelle menée auprès de 1945 étudiants de différentes facultés de l'Université Libanaise et d'autres universités privées. Un auto-questionnaire basé sur la méthode ASSIST (Alcohol, Smoking and Substance Involvement Screening Test) leur a été distribué. La prévalence de la consommation d'alcool au moins une fois au cours de la vie est de 20,9\%. Parmi les drogues, le cannabis (12,3\%) et les tranquillisants (11 \%) sont les plus utilisés tandis que la cocaïne $(3,3 \%)$ et les hallucinogènes $(3,6 \%)$ sont les moins utilisés. Le tabagisme à la cigarette et au narguilé, les sorties nocturnes, la pression des pairs et le manque d'activité durant le temps libre sont associés à la consommation problématique de substances, alors que la bonne relation avec les parents, le travail et la lecture en sont protecteurs. Il y a une prévalence élevée de consommation de substances psychoactives chez les étudiants universitaires au Liban. Une prise en charge pluridisciplinaire des étudiants dépendants est nécessaire pour répondre à la diversité de leurs besoins.

\section{Substance use among Lebanese university students: prevalence and associated factors}

ABSTRACT Scientific research on use and misuse of substances in Lebanon is scarce. This study aimed to evaluate the rate of use and abuse of substances among Lebanese youth and identify the determinants and risk factors behind these behaviours. An observational survey was conducted on 1945 university students selected from the different faculties of the Lebanese University and other private universities. A self-administered questionnaire based on ASSIST (Alcohol, Smoking and Substance Involvement Screening Test) was administered. The prevalence of ever consuming alcohol was $20.9 \%$. Cannabis (12.3\%) and tranquilizers (11\%) had the highest rates of ever use among the drugs, whereas cocaine (3.3\%) and hallucinogens $(3.6 \%)$ had the lowest rates. Smoking cigarettes and waterpipes, going out at night, peer pressure and having no specific leisure time activity were associated with problematic substance use, while a better relationship with parents, reading and working were inversely associated with use. There is a high prevalence of substance use among university students in Lebanon. Multidisciplinary support for addicted students is needed to meet their diverse needs.

'Laboratoire de recherche clinique et épidémiologique, Faculté de Pharmacie, Université Libanaise, Beyrouth (Liban) (Correspondance à adresser à P. Salameh : pascalesalameh@hotmail.com ; psalameh@ul.edu.lb). École doctorale des Sciences et de Technologie, Université Libanaise,

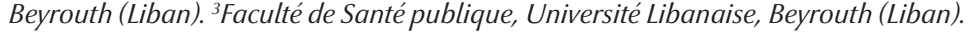

Reçu : 14/02/13; accepté : 08/12/13 


\section{Introduction}

Bien que l'histoire de la toxicomanie ait accompagné la découverte des substances psychoactives, les études épidémiologiques scientifiques datent à peine de trois décennies (1). La morbimortalité liée à ces substances parmi les adolescents est devenue une pandémie mondiale (2-10). Les adolescents de 18 à 25 ans sont les plus vulnérables, avec un pourcentage plus élevé pour la consommation hors prescription médicale (11), particulièrement de médicaments contre la douleur et de stimulants (12).

Ainsi la consommation sans prescription médicale de substances psychoactives constitue-t-elle une préoccupation et un problème de santé publique dans le monde entier (1315). Le tabac, l'alcool et les produits illicites figurent parmi les 20 premiers facteurs de risque pour la santé selon l'Organisation mondiale de la Santé (OMS). On estime que le tabac est responsable de 8,8\% de la mortalité et de $4,1 \%$ du fardeau global de toutes les maladies, mesuré par le nombre d'années de vie corrigées de l'incapacité (AVCI). Cependant, l'alcool est responsable de 3,2 \% de la mortalité et de $4 \%$ des AVCI. Les produits illicites, à leur tour, sont à l'origine de $0,4 \%$ de mortalité et de 0,8\% d'AVCI (16). La consommation excessive d'alcool ainsi que l'abus d'autres substances psychoactives sont aussi des facteurs de risque pour de multiples problèmes sociaux, financiers et légaux pour les individus et leur famille. Des études épidémiologiques ont montré une augmentation de la prévalence de la consommation de substances psychoactives $(11,14,17$ 19). Selon ces études, les substances les plus consommées sont la marijuana, suivie par les dérivés morphiniques $(11,20)$.

Les raisons qui poussent les jeunes à consommer de la drogue sont multiples, comprenant des facteurs génétiques, familiaux et sociaux. En outre, l'effet des copains, l'état de santé, le niveau d'études, l'usage par les parents et le début précoce de consommation de substances psychoactives sont des facteurs aussi importants liés au développement de l'abus et de la dépendance (21).

Les études déjà réalisées au Liban (22-29) n'ayant pas été menées sur des échantillons représentatifs de la population des étudiants universitaires libanais, notre objectif était d'évaluer le taux de consommation et d'abus de substances psychoactives auprès des jeunes Libanais et d'identifier les déterminants et les facteurs de risque essentiels derrière ces comportements.

\section{Méthodologie}

\section{Échantillonnage}

Il était difficile d'avoir accès aux campus universitaires pour faire remplir les questionnaires aux étudiants ou bien d'obtenir les listes complètes des étudiants par faculté et par université pour pouvoir tirer un échantillon aléatoire ; un échantillon par quotas proportionnel à la taille des universités a donc été sélectionné. L'étude a été menée entre février et juillet 2012. Dans les facultés des différentes universités publiques et privés, 2974 questionnaires ont été distribués. Le taux de réponse était de 65,4\% : 1945 étudiants, dont 987 ont répondu en arabe (50,75\%), $564(29,0 \%)$ en français et $394(20,25 \%)$ en anglais.

\section{Procédure}

Les étudiants ont été informés qu'il s'agissait d'une étude servant à évaluer leur consommation de substances psychoactives. Un consentement éclairé oral leur a été demandé avant de remplir le questionnaire, tout en leur assurant l'anonymat et le respect de la confidentialité de leurs réponses. Les questionnaires ont donc été distribués, auto-remplis par les étudiants dans un lieu tranquille loin de l'enquêteur et de leurs pairs, puis rendus à l'enquêteur et rangés dans des boîtes fermées. Le temps moyen pour remplir le questionnaire était de 15 minutes.

\section{Outil}

Un auto-questionnaire, préalablement traduit et validé par l'OMS (30), dans les trois langues (anglais, arabe et français), a été distribué. Le choix a été laissé à l'étudiant par rapport à la langue du questionnaire auquel il aimerait répondre. Le questionnaire est basé sur celui du test ASSIST (Alcohol, Smoking and Substance InvolvementScreening Test), version 3. Ce questionnaire permet un dépistagebrefmais extensifpourdiverses substances psychoactives : tabac, alcool, cannabis, cocaine, stimulants de type amphétamines, solvants, calmants ou somnifères, hallucinogènes, opiacés et autres drogues. De même, il permet d'objectiver et de quantifier la consommation de substances psychoactives et ses répercussions. Il propose, selon un nombre de points définis (scores), la suite de la prise en charge médicale : 0 à 3 points pas d'intervention ; 4 à 26 intervention brève; $\geq 27$ traitement plus intensif (par un médecin de premier recours ou un service spécialisé). À noter que les scores concernant l'alcool sont différents, avec une répartition respectivement de 0 à 10,11 à 26 et $\geq 27$. Sa sensibilité varie de 54 à $97 \%$ et sa spécificité de 50 à $96 \%$, selon la substance concernée (31).Lesindividus ayant besoin d'intervention (brève ou intensive) ont été considérés comme à consommation problématique ou à risque.

\section{Analyse statistique}

La taille de l'échantillon a été calculée sur Epi Info version 6, alors que les données ont été saisies et analysées sur SPSS version 20. Un test est considéré significatif pour $p<0,05$. Les tests statistiques appropriés ont été choisis en fonction de la nature 
des variables : le test $\mathrm{du} \chi^{2}$ pour les variables nominales, l'analyse de variance (ANOVA) et le test $t$ pour les variables continues, et la régression logistique pour expliquer la consommation problématique de substances psychoactives par les autres variables indépendantes, notamment l'âge, le sexe, la nationalité, la bonne relation avec les parents, la bonne relation avec les amis, la connaissance d'une personne qui utilise la substance en question, la façon de passer son temps libre (lecture, prière, sports, ennui, etc.), les sorties nocturnes et le tabagisme à la cigarette et au narguilé.

\section{Résultats}

\section{Caractéristiques de l'échantillon}

Les répondants comprenaient 850 étudiants des universités privées $(43,7 \%)$ et 1095 étudiants de l'Université Libanaise (UL) publique (56,3\%), formant un total de 1945 étudiants. Le tableau 1 présente les caractéristiques démographiques de l'échantillon. Les garçons constituent $46,2 \%$ et les filles $53,8 \%$. La majorité des étudiants sont âgés de 17 à 21 ans $(74,9 \%)$ et $87,7 \%$ sont de nationalité libanaise. Les étudiants des facultés de gestion et de droit constituent $32,1 \%$ de l'échantillon, suivis par ceux des facultés de génie et des arts (26,3\%), des sciences $(25,1 \%)$ et enfin des facultés médicales : pharmacie, médecine, dentaire et santé publique $(16,5 \%)$.

\section{Consommation de substances psychoactives selon le type d'université}

Dans le tableau 2, nous présentons les scores spécifiques pour chaque substance : il n'y a pas de différence significative entre les universités privées et l'UL, à l'exception de la consommation de stimulants qui parait être plus élevée parmi les étudiants de

\begin{tabular}{|c|c|c|}
\hline Caractéristiques & Nbre & $\%$ \\
\hline \multicolumn{3}{|l|}{ Sexe } \\
\hline Masculin & 898 & 46,2 \\
\hline Féminin & 1046 & 53,8 \\
\hline \multicolumn{3}{|l|}{$\hat{A g e}$ (ans) } \\
\hline $17-18$ & 605 & 31,1 \\
\hline $19-21$ & 852 & 43,8 \\
\hline$>21$ & 488 & 25,1 \\
\hline \multicolumn{3}{|l|}{ Nationalité } \\
\hline Libanaise & 1697 & 87,7 \\
\hline Étrangère & 237 & 12,3 \\
\hline \multicolumn{3}{|l|}{ Université } \\
\hline Libanaise & 1095 & 56,3 \\
\hline Privées & 850 & 43,7 \\
\hline American University of Beirut & 262 & 30,8 \\
\hline Lebanese American University & 45 & 5,3 \\
\hline Université Saint-Joseph & 83 & 9,8 \\
\hline Lebanese International University & 69 & 8,1 \\
\hline Notre Dame University & 39 & 4,6 \\
\hline Université Saint-Esprit de Kaslik & 37 & 4,3 \\
\hline Université La Sagesse & 16 & 1,9 \\
\hline Beirut Arab University & 141 & 16,6 \\
\hline Université Antonine & 20 & 2,4 \\
\hline Université de Balamand & 28 & 3,3 \\
\hline American University of Technology & 34 & 4,0 \\
\hline Autres & 76 & 8,9 \\
\hline \multicolumn{3}{|l|}{ Faculté } \\
\hline Gestion & 412 & 21,2 \\
\hline Droit & 212 & 10,9 \\
\hline Génie & 276 & 14,2 \\
\hline Arts & 236 & 12,1 \\
\hline Sciences & 489 & 25,1 \\
\hline Pharmacie & 132 & 6,8 \\
\hline Médecine & 57 & 2,9 \\
\hline Dentaire & 56 & 2,9 \\
\hline Santé publique & 75 & 3,9 \\
\hline
\end{tabular}

l'UL $(p=0,002)$. De plus, les étudiants ayant un score de $0-10$ pour l'alcool et de 0-3 pour les autres substances constituent la majorité, et ne nécessitent aucune intervention.

Un score de 11-26 pour l'alcool et de 4-26 pour les autres substances demandent une intervention brève auprès des étudiants ; le pourcentage pour ces tranches d'âge est substantiel. Cependant, un score $\geq 27$ impose une action rapide et un traitement plus intensif pour plus de $1 \%$ des étudiants pourl'alcool.

\section{Consommation de substances psychoactives selon le sexe}

Le tableau 3 montre que la prévalence de la consommation d'alcool au moinsunefois au cours delavieestélevée parmi les étudiants universitaires, avec une tendance plus forte chez les garçons 


\begin{tabular}{|c|c|c|c|c|c|}
\hline \multirow[t]{2}{*}{ Score ASSIST } & \multicolumn{2}{|c|}{$\begin{array}{l}\text { Université Libanaise } \\
\qquad(n=1095)\end{array}$} & \multicolumn{2}{|c|}{$\begin{array}{l}\text { Universités privées } \\
\qquad(n=850)\end{array}$} & \multirow[t]{2}{*}{$p$} \\
\hline & Nbre & $\%$ & Nbre & $\%$ & \\
\hline Consommateurs d'alcool ${ }^{a}$ & 436 & 39,8 & 401 & 47,2 & $<0,001$ \\
\hline $0-10$ & 258 & 23,6 & 304 & 35,8 & \\
\hline $11-26$ & 171 & 15,6 & 78 & 9,2 & \\
\hline$\geq 27$ & 7 & 0,6 & 19 & 2,2 & \\
\hline Utilisateurs de cannabis & 105 & 9,6 & 123 & 14,5 & 0,176 \\
\hline $0-3$ & 67 & 6,1 & 75 & 8,9 & \\
\hline $4-26$ & 32 & 2,9 & 46 & 5,4 & \\
\hline$\geq 27$ & 6 & 0,6 & 2 & 0,2 & \\
\hline Utilisateurs de cocaïne & 32 & 3,0 & 32 & 3,8 & 1,000 \\
\hline $0-3$ & 13 & 1,2 & 14 & 1,6 & \\
\hline $4-26$ & 15 & 1,4 & 15 & 1,8 & \\
\hline$\geq 27$ & 4 & 0,4 & 3 & 0,4 & \\
\hline Utilisateurs de stimulants & 126 & 11,5 & 57 & 6,7 & 0,002 \\
\hline $0-3$ & 90 & 8,2 & 30 & 3,5 & \\
\hline $4-26$ & 34 & 3,1 & 20 & 2,4 & \\
\hline$\geq 27$ & 2 & 0,2 & 7 & 0,8 & \\
\hline Utilisateurs de solvants & 111 & 10,2 & 26 & 3,0 & 0,080 \\
\hline $0-3$ & 93 & 8,5 & 17 & 2,0 & \\
\hline $4-26$ & 15 & 1,4 & 7 & 0,8 & \\
\hline$\geq 27$ & 3 & 0,3 & 2 & 0,2 & \\
\hline Utilisateurs de tranquillisants & 136 & 12,4 & 75 & 8,8 & 0,079 \\
\hline $0-3$ & 91 & 8,3 & 46 & 5,4 & \\
\hline $4-26$ & 33 & 3,0 & 27 & 3,2 & \\
\hline$\geq 27$ & 12 & 1,1 & 2 & 0,2 & \\
\hline Utilisateurs d'hallucinogènes & 46 & 4,3 & 26 & 3,0 & 0,442 \\
\hline $0-3$ & 29 & 2,7 & 14 & 1,6 & \\
\hline $4-26$ & 14 & 1,3 & 8 & 0,9 & \\
\hline$\geq 27$ & 3 & 0,3 & 4 & 0,5 & \\
\hline Utilisateurs d'opiacés & 43 & 4,0 & 31 & 3,7 & 0,542 \\
\hline $0-3$ & 25 & 2,3 & 21 & 2,5 & \\
\hline $4-26$ & 16 & 1,5 & 10 & 1,2 & \\
\hline$\geq 27$ & 2 & 0,2 & 0 & 0,0 & \\
\hline
\end{tabular}

ASSIST: Alcohol, Smoking and Substance Involvement Screening Test. ${ }^{a}$ Score spécifique.

à boire de l'alcool que chez les filles $(p<0,001)$. D’autre part, la prévalence la plus élevée de consommation de substances au moins une fois au cours de la vie, parmi les drogues, se trouve pour lecannabis (12,3\%) et les tranquillisants $(11 \%)$ alors que les drogues les moins utilisées par les étudiants universitaires paraissent être la cocaïne (3,3\%) et les hallucinogènes $(3,6 \%)$.
Une différence significative existe entre les deux sexes, les garçons consommant les substances suivantes le plus fréquemment (à l'exception des tranquillisants) : cannabis (garçons $16,9 \%$, filles $8,5 \% ; p<0,001)$, cocaïne (garçons $5,2 \%$, filles $1,8 \%$; $p<0,001$ ), stimulants (garçons $12,6 \%$, filles $4,8 \% ; p<0,001)$, solvants (garçons $9,1 \%$, filles $4,7 \%$; $p=0,001$ ), hallucinogènes (garçons
$5,7 \%$, filles $1,8 \% ; p<0,001)$, opiacés (garçons 5,8 \%, filles 2,0 \% ; $p<0,001)$. De plus, les résultats montrent que $36,3 \%$ des étudiants universitaires ont fumé au moins une fois au cours de leur vie avec des prévalences largement différentes entre les deux sexes, les garçons fumant beaucoup plus que les filles (garçons $49,1 \%$, filles $25,5 \%$; $p<0,001$ ) (Tableau 3). 


\begin{tabular}{|c|c|c|c|c|c|c|c|}
\hline \multirow[t]{2}{*}{ Type de substance } & \multicolumn{2}{|c|}{$\begin{array}{l}\text { Sexe masculin } \\
\quad(n=898)\end{array}$} & \multicolumn{2}{|c|}{$\begin{array}{l}\text { Sexe féminin } \\
\quad(n=1046)\end{array}$} & \multicolumn{2}{|c|}{$\begin{array}{c}\text { Total } \\
(n=1945)\end{array}$} & \multirow[t]{2}{*}{$p$} \\
\hline & Nbre & $\%$ & Nbre & $\%$ & Nbre & $\%$ & \\
\hline Alcool & 530 & 59,0 & 398 & 38,0 & 928 & 47,7 & $<0,001$ \\
\hline Cannabis & 151 & 16,9 & 89 & 8,5 & 240 & 12,3 & $<0,001$ \\
\hline Cocaïne & 46 & 5,2 & 18 & 1,8 & 64 & 3,3 & $<0,001$ \\
\hline Stimulants & 112 & 12,6 & 50 & 4,8 & 162 & 8,3 & $<0,001$ \\
\hline Solvants & 81 & 9,1 & 49 & 4,7 & 130 & 6,7 & 0,001 \\
\hline Tranquillisants & 98 & 11,0 & 116 & 11,1 & 214 & 11,0 & 0,910 \\
\hline Hallucinogènes & 51 & 5,7 & 19 & 1,8 & 70 & 3,6 & $<0,001$ \\
\hline Opiacés & 52 & 5,8 & 21 & 2,0 & 73 & 3,8 & $<0,001$ \\
\hline Tabac & 441 & 49,1 & 266 & 25,5 & 707 & 36,3 & $<0,001$ \\
\hline
\end{tabular}

\section{Consommation de substances psychoactives par groupe d'âge}

La consommation d'alcool ne diffère pas significativement selon la tranche d'âge (Tableau 4). D'autre part, on remarque une augmentation de la consommation du cannabis et de la cocaïne avec l'âge : cannabis (1718 ans 8,9\%, 19-21 ans 10,2\%, $>21$ ans $20,3 \% ; p<0,001)$, cocaïne (17-18 ans $3,0 \%$, $19-21$ ans $1,9 \%$, > 21 ans $6,1 \%$; $p<0,001)$. De même en ce qui concerne le tabac, plus l'âge est élevé, plus la prévalence du tabagisme augmente : $17-18$ ans $31,7 \%$, $19-21$ ans $35,6 \%,>21$ ans $43,7 \%$; $p<0,001$ (Tableau 4).

\section{Fréquence de consommation de substances psychoactives au cours des trois derniers mois}

Une proportion de $14,8 \%$ des étudiants rapporte avoir bu de l'alcool hebdomadairement au cours des trois derniers mois et 3,0 \% presque chaque jour, avec une prévalence plus élevée chez les garçons que chez les filles chaque jour ou presque chaque jour $(p<0,001)$. Une portion non négligeable d'étudiants déclare leur consommation hebdomadaire ou même journalière d'autres substances, respectivement: cannabis $(0,9 \%, 0,5 \%)$, cocaïne $(0,2 \%$, $0,4 \%)$, stimulants $(1,0 \%$, $0,3 \%)$, solvants $(0,3 \%, 0,05 \%)$, calmants $(0,7 \%, \quad 0,7 \%)$, hallucinogènes $(0,1 \%, 0,1 \%)$, opiacés $(0,1 \%, 0,3 \%)$ (Tableau 5$)$. De plus, 4,3\% des étudiants fument plus de 20 cigarettes par jour et $6,9 \%$ fument 10-20 cigarettes/jour alors que $3,9 \%$ fument moins de 6 narguilés par semaine et $7,7 \%$ fument 2-6 narguilés/semaine.

\section{Analyses multivariées}

Les facteurs qui semblent affecter le score spécifique pour chaque substance ont été introduits dans une analyse de régression linéaire pour identifier ceux qui sont les plus prédictifs (Tableau 6).

En somme, le sexe féminin, l'âge plus avancé, une bonne relation avec les parents, la lecture et le

\begin{tabular}{|c|c|c|c|c|c|c|c|}
\hline \multirow[t]{3}{*}{ Type de substance } & \multicolumn{6}{|c|}{ Groupe d'âge (ans) } & \multirow[t]{3}{*}{$p$} \\
\hline & \multicolumn{2}{|c|}{$\begin{array}{c}17-18 \\
(n=605)\end{array}$} & \multicolumn{2}{|c|}{$\begin{array}{c}19-21 \\
(n=852)\end{array}$} & \multicolumn{2}{|c|}{$\begin{array}{c}>21 \\
(n=488)\end{array}$} & \\
\hline & Nbre & $\%$ & Nbre & $\%$ & Nbre & $\%$ & \\
\hline Alcool & 278 & 46,0 & 399 & 46,8 & 207 & 42,4 & 0,283 \\
\hline Cannabis & 54 & 8,9 & 87 & 10,2 & 99 & 20,3 & $<0,001$ \\
\hline Cocaïne & 18 & 3,0 & 16 & 1,9 & 30 & 6,1 & $<0,001$ \\
\hline Stimulants & 45 & 7,4 & 69 & 8,1 & 48 & 9,9 & 0,543 \\
\hline Solvants & 39 & 6,4 & 52 & 6,1 & 39 & 8,0 & 0,421 \\
\hline Tranquillisants & 74 & 12,2 & 79 & 9,3 & 61 & 12,5 & 0,059 \\
\hline Hallucinogènes & 21 & 3,5 & 27 & 3,2 & 22 & 4,5 & 0,754 \\
\hline Opiacés & 16 & 2,7 & 34 & 4,0 & 23 & 4,7 & 0,185 \\
\hline Tabac & 192 & 31,7 & 303 & 35,6 & 213 & 43,7 & $<0,001$ \\
\hline
\end{tabular}




\begin{tabular}{|c|c|c|c|c|c|c|c|c|c|c|}
\hline \multirow[t]{2}{*}{ Type de substance } & \multicolumn{2}{|c|}{ Jamais } & \multicolumn{2}{|c|}{$1-2$ fois } & \multicolumn{2}{|c|}{ Mensuelle } & \multicolumn{2}{|c|}{ Hebdomadaire } & \multicolumn{2}{|c|}{$\begin{array}{c}\text { Chaque jour ou } \\
\text { presque }\end{array}$} \\
\hline & Nbre & $\%$ & Nbre & $\%$ & Nbre & $\%$ & Nbre & $\%$ & Nbre & $\%$ \\
\hline Alcool & 144 & 7,4 & 160 & 8,2 & 152 & 7,8 & 288 & 14,8 & 58 & 3,0 \\
\hline Cannabis & 128 & 6,6 & 53 & 2,7 & 21 & 1,1 & 18 & 0,9 & 10 & 0,5 \\
\hline Cocaïne & 34 & 1,7 & 9 & 0,5 & 11 & 0,6 & 3 & 0,2 & 7 & 0,4 \\
\hline Stimulants & 116 & 6,0 & 28 & 1,4 & 16 & 0,8 & 19 & 1,0 & 5 & 0,3 \\
\hline Solvants & 108 & 5,6 & 15 & 0,8 & 8 & 0,4 & 5 & 0,3 & 1 & 0,05 \\
\hline Tranquillisants & 139 & 7,1 & 31 & 1,6 & 15 & 0,8 & 13 & 0,7 & 14 & 0,7 \\
\hline Hallucinogènes & 47 & 2,4 & 10 & 0,5 & 10 & 0,5 & 2 & 0,1 & 2 & 0,1 \\
\hline Opiacés & 48 & 2,5 & 8 & 0,4 & 11 & 0,6 & 2 & 0,1 & 5 & 0,3 \\
\hline
\end{tabular}

travail sont inversement associés à la consommation de substances. Le fait de passer son temps libre à dormir ou à s'ennuyer, fumer des cigarettes ou le narguilé, et surtout la connaissance de consommateurs de substances sont significativement associés à la consommation problématique de substances; le sport est aussi associé à la consommation de stimulants.

\section{Discussion}

La prévalence de la consommation de substances psychoactives parmi les étudiants universitaires au Liban est alarmante. En comparant avec la dernière étude réalisée par Karam et al. en 2000 (22), nous remarquons qu'entre les années 2000 et 2012, la consommation d'opiacés a légèrement diminué de $4,5 \%$ à $3,8 \%$ alors que la consommation de cannabis a augmenté, passant de $2,6 \%$ à $12,3 \%$, celle de cocaïne de $0,5 \%$ à $3,3 \%$, de stimulants de $1,8 \%$ à $8,3 \%$, de tranquillisants de $10,2 \%$ à $11,0 \%$ et finalement de tabac de $18,3 \%$ à $36,3 \%$. Seule la consommation d'alcool a diminué passant de $49,7 \%$ à $20,9 \%$ : ceci pourrait être dû aux différences au niveau de l'échantillonnage, notre étude étant menée sur un échantillon plus large que celle de Karam et al., qui ne prenait pas en compte l'UL.
Malgré les problèmes sociaux, économiques et politiques au Liban, le manque de contrôle sur les prescriptions médicales et l'accès facile aux médicaments (22), nos résultats restent toujours inférieurs à ceux obtenus dans les pays développés, par exemple ceux du National Institute on Drug Abuse (NIDA) [Institut national sur l'abus de drogues] aux États-Unis : ce dernier a signalé une prévalence de la consommation d'alcool de $94,1 \%$, de stimulants de $10,5 \%$, de cocaine de 7,9\% et d'opiacés de 7,3\% parmi les étudiants américains (32). Cette différence est probablement due aux liens familiaux et sociaux puissants au sein de notre société, ce qui joue un rôle assez important dans la protection des adolescents contre les comportements déviants (22) ; ceci a été retrouvé dans nos résultats.

Une étude conduite en mai 2010 (Ghandour et al.) sur 570 étudiants de l'université américaine de Beyrouth (AUB American University of Beirut] a conclu qu'au Liban, comme dans les autres cultures occidentales, une proportion considérable de jeunes ont tendance à l'automédication. Les prévalences d'usage médical et non médical des médicaments sont, respectivement, pour les douleurs de $36,9 \%$ et $15,1 \%$, pour l'anxiété de $8,3 \%$ et $4,6 \%$, pour le sommeil de $6,5 \%$ et $5,8 \%$ et pour les stimulants de $2,6 \%$ et 3,5\% (25).
L'usage des benzodiazépines est particulièrement élevé surtout chez les sujets âgés de plus de 45 ans, chez les femmes, chez les fumeurs et chez ceux ayant été confronté à un évènement récent (28).

L'analyse du score ASSIST permet d'évaluer la situation des étudiants universitaires libanais : il n'y a pas de différence entre les universités (privées et publiques) concernant les interventions nécessaires selon les scores spécifiques de chaque substance psychoactive, à l'exception de l'alcool qui est plus consommé dans les universités privées et des solvants qui semblent être plus consommés parmi les étudiants de l'UL. Une étude plus approfondie de l'influence du niveau socioéconomique des étudiants sur la consommation de substances psychoactives serait nécessaire pour expliquer ces résultats.

Bien qu'il soit difficile de mesurer l'ampleur de la toxicomanie au Liban, nous remarquons que de plus en plus de jeunes sont concernés (2225). L'étude la plus ancienne est celle réalisée par Nassar et al. en 1973 sur l'usage non médical de substances psychoactives (marijuana, LSD et amphétamine) par les étudiants de l'université américaine de Beyrouth (AUB). Sur un échantillon de 436 étudiants, environ $17 \%$ ont essayé la marijuana, $17 \%$ les 


\begin{tabular}{|c|c|c|c|c|c|}
\hline Variable dépendante & $\mathbf{R}^{2}$ & $\begin{array}{l}\text { Variables indépendantes retenues dans le } \\
\text { modèle final }\end{array}$ & $p$ & OR ajusté & IC $95 \%$ \\
\hline \multirow[t]{4}{*}{ Alcool } & \multirow[t]{4}{*}{0,367} & Sexe féminin & $<0,001$ & 0,33 & $0,19-0,59$ \\
\hline & & Bonne relation avec les parents & $<0,001$ & 0,32 & $0,18-0,57$ \\
\hline & & Nombre de cigarettes par jour & $<0,001$ & 1,62 & $1,40-1,87$ \\
\hline & & Passer son temps libre à dormir & 0,006 & 2,20 & $1,26-3,86$ \\
\hline \multirow[t]{3}{*}{ Cannabis } & \multirow[t]{3}{*}{0,449} & Bonne relation avec les parents & 0,024 & 0,40 & $0,18-0,86$ \\
\hline & & Nombre de cigarettes par jour & $<0,001$ & 1,65 & $1,35-2,01$ \\
\hline & & Nombre de narguilés par semaine & 0,006 & 1,36 & $1,09-1,69$ \\
\hline \multirow[t]{4}{*}{ Cocaïne } & \multirow[t]{4}{*}{0,306} & Sexe féminin & 0,056 & 0,30 & $0,09-1,03$ \\
\hline & & Âge plus avancé & 0,027 & 0,48 & $0,25-0,92$ \\
\hline & & Nombre de cigarettes par jour & 0,072 & 1,28 & $0,98-1,68$ \\
\hline & & Connaissance d'un consommateur & $<0,001$ & 17,54 & $4,95-62,50$ \\
\hline \multirow[t]{6}{*}{ Stimulants } & \multirow[t]{6}{*}{0,452} & Nombre de cigarettes par jour & 0,025 & 1,36 & $1,04-1,79$ \\
\hline & & Nombre de narguilés par semaine & 0,001 & 1,53 & $1,20-1,96$ \\
\hline & & Connaissance d'un consommateur & $<0,001$ & 0,04 & $0,01-0,15$ \\
\hline & & Passer son temps libre à s'ennuyer & 0,031 & 1,76 & $1,05-2,94$ \\
\hline & & Faire du sport & 0,006 & 4,21 & $1,52-11,68$ \\
\hline & & Lire & 0,072 & 0,21 & $0,039-1,15$ \\
\hline \multirow{3}{*}{$\begin{array}{l}\text { Substances inhalées } \\
\text { (colle, etc.) }\end{array}$} & \multirow[t]{3}{*}{0,666} & Travailler & 0,037 & 0,03 & $0,001-0,82$ \\
\hline & & Nombre de cigarettes par jour & 0,044 & 1,60 & $1,01-2,53$ \\
\hline & & Bonne relation avec les amis & 0,041 & 0,16 & $0,03-0,92$ \\
\hline \multirow[t]{2}{*}{ Hallucinogènes } & \multirow[t]{2}{*}{0,697} & Bonne relation avec les parents & 0,017 & 0,13 & $0,02-0,69$ \\
\hline & & Travailler & 0,006 & 0,06 & $0,01-0,45$ \\
\hline \multirow[t]{2}{*}{ Opioïdes } & \multirow[t]{2}{*}{0,579} & Nombre de cigarettes par jour & 0,009 & 1,79 & $1,16-2,77$ \\
\hline & & Passer son temps libre à s'ennuyer & 0,008 & 2,90 & $1,33-6,33$ \\
\hline \multirow{9}{*}{$\begin{array}{l}\text { Consommation } \\
\text { problématique } \\
\text { d'une substance } \\
\text { psychoactive au } \\
\text { moins }\end{array}$} & \multirow[t]{9}{*}{0,245} & Sexe féminin & $<0,001$ & 0,38 & $0,24-0,60$ \\
\hline & & Âge plus avancé & 0,069 & 0,76 & $0,56-1,02$ \\
\hline & & Bonne relation avec les parents & $<0,001$ & 0,38 & $0,23-0,65$ \\
\hline & & Nationalité non libanaise & 0,097 & 1,54 & $0,93-2,57$ \\
\hline & & Nombre de soirées par semaine & 0,159 & 1,14 & $0,95-1,36$ \\
\hline & & Nombre de cigarettes par jour & $<0,001$ & 1,38 & $1,20-1,58$ \\
\hline & & Nombre de narguilés par semaine & 0,031 & 1,18 & $1,02-1,36$ \\
\hline & & Bonne relation avec les amis & 0,065 & 1,80 & $0,96-3,38$ \\
\hline & & Passe son temps libre à dormir & 0,197 & 1,34 & $0,86-2,10$ \\
\hline
\end{tabular}

OR: odds ratio ; IC : intervalle de confiance.

tranquillisants, $15 \%$ les somnifères, $8 \%$ les amphétamines et $2 \%$ les hallucinogènes type LSD (26). Une autre étude réalisée par la LFPA (Lebanese Family Planning Association) sur la dépendance aux drogues en 1995 parmi les étudiants universitaires au Liban $(n=3180)$ a conclu que le cannabis était le plus utilisé parmi toutes les substances psychoactives (0,9-3,2\%) (27). D'autre part, Karam et al. (2000) ont cherché à examiner les motifs de consommation des substances psychoactives dans deux universités privées (AUB et USJ). La prévalence des fumeurs était de 18,3\% alors que $49,4 \%$ ont consommé de l'alcool au moins une fois au cours de leur vie. De même, 2,1 \% abusent de l'alcoolet $2,4 \%$ sontalcoolo-dépendants selon le DSM-III (Diagnostic and Statistical Manual of Mental Disorders, 3rd edition [Manuel diagnostique et statistique des troubles mentaux, $3^{e}$ édition]). Les tranquillisants ont été les plus utilisés $(10,2 \%)$ et l'héroïne la substance la moins utilisée $(0,4 \%)$. Le taux d'abus et de dépendance pour ces substances (autres que l'alcool et le tabac) varie entre 0,1 et $0,8 \%(22)$.

En 2010, une évaluation rapide de la situation a été effectuée dans le but d'examiner la consommation et l'abus 
de substances psychoactives au Liban : $12 \%$ des étudiants au lycée fument au moins un paquet de cigarettes par jour et $9 \%$ des étudiants universitaires remplissent les critères d'abus d'alcool du DSM-IV (Manuel diagnostique et statistique des troubles mentaux, quatrième édition). Le cannabis figure en tête de liste des substances illicites consommées et les tranquillisants sont les substances les plus fréquemment utilisées parmi les jeunes lycéens et universitaires. L'héroïne est responsable de $50 \%$ des admissions en traitement, suivie par la cocaine $(20 \%)$ et l'alcool (20\%) (24).

Notre étude a aussi montré que le cannabis est la substance la plus fréquemment consommée après l'alcool, alors que la cocainne est la substance la moins consommée parmi les étudiants, à la différence des résultats obtenus dans une étude précédente (22), qui citait que les tranquillisants étaient les plus utilisés. De plus, nous avons trouvé que les garçons utilisent les substances psychoactives plus que les filles, ce qui coïncide avec les résultats trouvés par plusieurs chercheurs de la région (33-35), mais contredit à d'autres études qui n'ont pas trouvé ce rapport selon le sexe $(22,25)$.

Concernant le tabac, une étude a été menéeparTamimetal.surunéchantillon d'étudiants des universités publiques et privées : la moyenne d'âge des étudiants était de 21 ans et laprévalence globale du tabagisme (cigarettes et/ ou narguilé) était de $40 \%$ (29). Nous avons aussi trouvé, de façon similaire à d'autres études, que le tabagisme à la cigarette était systématiquement et quantitativement impliqué dans l'augmentation de la consommation problématique de plusieurs substances psychoactives $(36,37)$; par rapport au narguilé, le résultat que nous avons trouvé est le premier de son genre, à notre connaissance. Étant donné la vogue actuelle de tabagisme au narguilé au Liban et dans le monde, ce résultat nous semble alarmant et mérite des investigations plus approfondies. De plus, le nombre de soirées passées à l'extérieur de la maison $(38,39)$, la connaissance d'un consommateur d'une substance donnée $(40,41)$ et le fait de ne pas avoir d'activité spécifique durant le temps libre (dormir, s'ennuyer) avaient aussi un effet sur l'augmentation de la consommation problématique d'au moins une substance psychoactive $(42,43)$. En outre, le sport était surtout associé à la consommation de stimulants, ce qui a déjà été retrouvé dans plusieurs études au niveau international (4446). Par contre, une bonne relation avec les parents $(40,47,48)$, la lecture et le travail (reflétant l'aspiration d'éducation) en étaient protecteurs (44).

Comme toute étude observationnelle, des limites contrebalancent nos résultats. Plusieurs biais, en relation avec la méthodologie de notre étude, peuvent affecter nos résultats, notamment le biais de sélection puisque nous n'avons pas pu obtenir des listes complètes des étudiants dans chaque établissement universitaire. Nos résultats peuvent aussi être biaisés par l'absentéisme des toxicomanes, et donc une sousestimation de la prévalence réelle de la consommation de chaque substance. De plus, il n'est pas possible de garantir que chaque étudiant a rempli un seul questionnaire, étant donné l'anonymat à respecter; cependant, compte tenu du temps nécessaire pour remplir le questionnaire et de l'effort requis de la part du répondant, qui n'est pas récompensé, nous éloignons la probabilité qu'une seule personne le remplisse deux fois. D'autre part, nous avons utilisé le statut public/ privé pour évaluer le niveau socioéconomique, ayant présumé que les jeunes étudiants seraient hésitants ou n'auraient peut-être pas une idée claire des salaires mensuels de leurs parents. De cette façon, nous avons pu diminuer le biais d'information concernant cette variable. Le biais d'information est aussi une source supplémentaire de sousestimation des consommateurs de ces substances, puisque nous nous sommes basés sur un questionnaire sujet au biais de rappel et à la sous-déclaration de la consommation. Nous suggérons donc des études prospectives avec dosage des substances psychoactives au niveau des fluides biologiques pour surmonter ces problèmes.

\section{Conclusion}

Nos résultats montrent des chiffres substantiels d'étudiants universitaires qui auraient besoin d'interventions au niveau de la prévention de la consommation de substances psychoactives chez les garçons, et les plus âgés en particulier. Une bonne relation avec les parents semble protectrice, alors que les soirées hors domicile, le fait de connaitre quelqu'un qui consomme des substances psychoactives et le tabagisme favorisent ce type de comportement. Conflit d'intérêt : aucun.

\section{Soutien financier : aucun..}

\section{Références}

1. RussellJM, Newman SC, Bland RC. Epidemiology of psychiatric disorders in Edmonton. Drug abuse and dependence. Acta Psychiatr Scand Suppl. 1994;376 suppl 376:54-62. PMID:8178686
2. Weinberg NZ, Rahdert E, Colliver JD, Glantz MD. Adolescent substance abuse: a review of the past 10 years. J Am Acad Child Adolesc Psychiatry. 1998 Mar;37(3):252-61. PMID:9519629 
3. Burke JD Jr, Burke KC, Rae DS. Increased rates of drug abuse and dependence after onset of mood or anxiety disorders in adolescence. Hosp Community Psychiatry. 1994 May;45(5):451-5. PMID:8045539

4. Milgram GG. Adolescents, alcohol and aggression. J Stud Alcohol Suppl. 1993 Sep;11:53-61. PMID:8410964

5. Robins LN, Przybeck TR. Age of onset of drug use as a factor in drug and other disorders. NIDA Res Monogr. 1985;56:17892. PMID:3929100

6. Okasha A. Young people and the struggle against drug abuse in the Arab countries. Bull Narc. 1985 Apr-Sep;37(2-3):67-73. PMID:3878175

7. Alfaro Murillo E. Drug abuse in Costa Rica: a review of several studies. Bull Pan Am Health Organ. 1990;24(1):30-4. PMID:2331555

8. Kandel DB, Davies M. High school students who use crack and other drugs. Arch Gen Psychiatry. 1996 Jan;53(1):71-80. PMID:8540779

9. Wright JD, Pearl L. Knowledge and experience of young people regarding drug misuse, 1969-94. BMJ. 1995 Jan 7;310(6971):20-4. PMID:7755714

10. Chen K, Kandel DB. The natural history of drug use from adolescence to the mid-thirties in a general population sample. Am J Public Health. 1995 Jan;85(1):41-7. PMID:7832260

11. Blanco C, Alderson D, Ogburn E, Grant BF, Nunes EV, Hatzenbuehler ML, et al. Changes in the prevalence of nonmedical prescription drug use and drug use disorders in the United States: 1991-1992 and 2001-2002. Drug Alcohol Depend. 2007 Oct 8;90(2-3):252-60. PMID:17513069

12. Compton WM, Volkow ND. Major increases in opioid analgesic abuse in the United States: concerns and strategies. Drug Alcohol Depend. 2006 Feb 1;81(2):103-7. PMID:16023304

13. Friedman RA. The changing face of teenage drug abuse-the trend toward prescription drugs. N Engl J Med. 2006 Apr 6;354(14):1448-50. PMID:16598042

14. Kuehn BM. Shift seen in patterns of drug use among teens. JAMA. 2006 Feb 8;295(6):612-3. PMID:16467223

15. Zarocostas J. Misuse of prescription drugs could soon exceed that of illicit narcotics, UN panel warns. BMJ. 2007 Mar 3;334(7591):444. PMID:17332555

16. Babor T, de la Fuente J, Saunders J, Grant M. AUDIT, The Alcohol Use Disorders Identification Test: Guidelines for use in primary health care. Geneva: World Health Organisation; 1989.

17. Figueiras A, Caamaño F, Gestal-Otero JJ. Sociodemographic factors related to self-medication in Spain. Eur J Epidemiol. 2000 Jan;16(1):19-26. PMID:10780338

18. Haydon E, Rehm J, Fischer B, Monga N, Adlaf E. Prescription drug abuse in Canada and the diversion of prescription drugs into the illicit drug market. Can J Public Health. 2005 NovDec;96(6):459-61. PMID:16350874

19. Johnston LD. Prescription drug use by adolescents: what we are learning and what we still need to know. J Adolesc Health. 2009 Dec;45(6):539-40. PMID:19931823

20. Catalano RF, White HR, Fleming CB, Haggerty KP. Is nonmedical prescription opiate use a unique form of illicit drug use? Addict Behav. 2011 Jan-Feb;36(1-2):79-86. PMID:20864261

21. Newcomb MD, Maddahian E, Bentler PM. Risk factors for drug use among adolescents: concurrent and longitudinal analyses. Am J Public Health. 1986 May;76(5):525-31. PMID:3485928
22. Karam E, Melhem N, Mansour C, Maalouf W, Saliba S, Chami A. Use and abuse of licit and illicit substances: prevalence and risk factors among students in Lebanon. Eur Addict Res. 2000 Dec;6(4):189-97. PMID:11124572

23. Karam EG, Maalouf WE, Ghandour LA. Alcohol use among university students in Lebanon: prevalence, trends and covariates. The IDRAC University Substance Use Monitoring Study (1991 and 1999). Drug Alcohol Depend. 2004 Dec 7;76(3):273-86. PMID:15561478

24. Karam EG, Ghandour LA, Maalouf WE, Yamout K, Salamoun MM. A rapid situation assessment (RSA) study of alcohol and drug use in Lebanon. J Med Liban. 2010 Apr-Jun;58(2):76-85. PMID:20549893

25. Ghandour LA, El Sayed DS, Martins SS. Prevalence and patterns of commonly abused psychoactive prescription drugs in a sample of university students from Lebanon: an opportunity for cross-cultural comparisons. Drug Alcohol Depend. 2012 Feb 1;121(1-2):110-7. PMID:21924844

26. Nassar NT, Melikian LH, Der-Karabetian A. Studies in the non medical use of drugs in Lebanon. I. The non medical use of marijuana, LSD, and amphetamine by students at the American University of Beirut. J Med Liban. 1973;26(3):21532. PMID:4759645

27. Lebanese Family Planning Association: Against Addiction. Beirut, Lebanese Family Planning Association.

28. Naja WJ, Pelissolo A, Haddad RS, Baddoura R, Baddoura C. A general population survey on patterns of benzodiazepine use and dependence in Lebanon. Acta Psychiatr Scand 2000 Dec;102(6):429-31 PMID:11142431.

29. Tamim H, Terro A, Kassem H, Ghazi A, Khamis TA, Hay MM, et al. Tobacco use by university students, Lebanon, 2001. Addiction. 2003 Jul;98(7):933-9. PMID:12814499

30. Sue Henry-Edwards. Rachel Humeniuk, Robert Ali, Vladimir Poznyakand Maristela Monteiro. The Alcohol, Smoking and Substance Involvement Screening Test (ASSIST): Guidelines for Use in Primary Care (Draft Version 1.1 for Field Testing). Geneva: World HealthOrganization; 2003.

31. Humeniuk R, Ali R, Babor TF, Farrell M, Formigoni ML, Jittiwutikarn J, et al. Validation of the alcohol, smoking and substance involvement screening test (ASSIST). Addiction. 2008 Jun;103(6):1039-47. PMID:18373724

32. Johnston L, O'Malley P, Bachman J. College students and young adults: NIH publication 93-3598. National Survey Results on Drug Use from the Monitoring the Future Study, 1975-1992. Volume 2. Rockville (Md.): National Institute on Drug Abuse; 1993.

33. Momtazi S, Rawson R. Substance abuse among Iranian high school students. Curr Opin Psychiatry. 2010 May;23(3):221-6. PMID:20308905

34. Ilhan IO, Yildirim F, Demirbaş H, Doğan YB. Prevalence and sociodemographic correlates of substance use in a university-student sample in Turkey. Int J Public Health. 2009;54(1):40-4. PMID:19142577

35. Refaat A. Practice and awareness of health risk behaviour among Egyptian university students. East Mediterr Health J. 2004 Jan-Mar;10(1-2):72-81. PMID:16201711

36. Saingam D, Assanangkornchai S, Geater AF. Drinking-smoking status and health risk behaviors among high school students in Thailand. J Drug Educ. 2012;42(2):177-93. PMID:23185837

37. Paksi B, Arnold P, Kun B, Demetrovics Z. [Association of the different types of substance use behaviors in the Hungarian adult population]. Psychiatr Hung. 2011;26(4):258-66. PMID:22058258

38. Viana M, Postigo C, Querol X, Alastuey A, López de Alda MJ, Barceló D, et al. Cocaine and other illicit drugs in 
airborne particulates in urban environments: a reflection of social conduct and population size. Environ Pollut. 2011 May;159(5):1241-7. PMID:21330024

39. Bellis MA, Hughes K. Getting drunk safely? Night-life policy in the UK and its public health consequences. Drug Alcohol Rev. 2011 Sep;30(5):536-45. PMID:21896076

40. Scherrer JF, Xian H, Pan H, Pergadia ML, Madden PA, Grant JD, et al. Parent, sibling and peer influences on smoking initiation, regular smoking and nicotine dependence. Results from a genetically informative design. Addict Behav. 2012 Mar;37(3):240-7. PMID:22094168

41. Geramian N, Akhavan S, Gharaat L, Tehrani AM, Farajzadegan Z. Determinants of drug abuse in high school students and their related knowledge and attitude. J Pak Med Assoc. 2012 Mar;62(3) Suppl 2:S62-6. PMID:22768463

42. Peltzer K. Leisure time physical activity and sedentary behavior and substance use among in-school adolescents in eight African countries. Int J Behav Med. 2010 Dec;17(4):2718. PMID:20054676

43. Fauth RC, Roth JL, Brooks-Gunn J. Does the neighborhood context alter the link between youth's after-school time activities and developmental outcomes? A multilevel analysis. Dev Psychol. 2007 May;43(3):760-77. PMID:17484586
44. VuoriMT, Kannas LK, Villberg J, Ojala SA, TynjäläJA, Välimaa RS. Is physical activity associated with low-risk health behaviours among 15-year-old adolescents in Finland? Scand J Public Health. 2012 Feb;40(1):61-8. PMID:22042742

45. Terry-McElrath YM, O'Malley PM, Johnston LD. Exercise and substance use among American youth, 1991-2009. Am J Prev Med. 2011 May;40(5):530-40. PMID:21496752

46. Sekulic D, Ostojic M, Ostojic Z, Hajdarevic B, Ostojic L. Substance abuse prevalence and its relation to scholastic achievement and sport factors: an analysis among adolescents of the Herzegovina-Neretva Canton in Bosnia and Herzegovina. BMC Public Health. 2012;12:274. PMID:22480230

47. Brooks FM, Magnusson J, Spencer N, Morgan A. Adolescent multiple risk behaviour: an asset approach to the role of family, school and community. J Public Health (Oxf). 2012 Mar;34 Suppl 1:i48-56. PMID:22363031

48. Malta DC, Porto DL, Melo FC, Monteiro RA, Sardinha LM, Lessa $\mathrm{BH}$. Family and the protection from use of tobacco, alcohol, and drugs in adolescents, National School Health Survey. Rev Bras Epidemiol. 2011 Sep;14 Suppl 1:166-77. PMID:22002153 